\title{
TESTE DE IMPACTO IZOD EM COMPÓSITOS DE MATRIZ POLIÉSTER REFORÇADOS COM FIBRAS DE CÂNHAMO*
}

\author{
Dhyemila de Paula Mantovani ${ }^{1}$ \\ Janaina da Silva Vieira ${ }^{1}$ \\ Anna Carolina Cerqueira Neves ${ }^{1}$ \\ Lucas de Almeida Pontes ${ }^{1}$ \\ Lázaro Araújo Rohen ${ }^{1}$ \\ Frederico Muylaert Margem ${ }^{2}$ \\ Felipe Perissé Duarte Lopes ${ }^{1}$ \\ Carlos Mauricio Fontes Vieira ${ }^{1}$ \\ Sergio Neves Monteiro ${ }^{3}$
}

\section{Resumo}

Hoje em dia, a necessidade de utilizar materiais ecologicamente corretos tem aumentando em paralelo com a constante busca de novas tecnologias para produzir materiais renováveis, economicamente viáveis e tecnologicamente avançados, o que justifica esse trabalho. $O$ presente trabalho consiste em estudar 0 comportamento mecânico de compósitos de matriz poliéster reforçados com fibras de cânhamo e testadas com impacto Izod. Para a realização deste teste, foram feitas amostras com dimensões de $10 \mathrm{~mm}$ de largura, $63 \mathrm{~mm}$ de comprimento e 12,7 $\mathrm{mm}$ de espessura em diferentes frações volumétricas de fibra, $0 \%, 10 \%, 20 \%$ e $30 \%$. As amostras foram testadas em um conjunto pendular PANTEC na configuração Izod, modelo XC-50 localizado no LAMAV/UENF. A energia de impacto Izod dos compósitos de poliéster de 30\% de fibras de cânhamo foi de 169,4 J/m.

Palavras-chave: Teste de Impacto Izod; Poliéster; Fibra de Cânhamo.

\section{IZOD IMPACT TESTS OF POLYESTER MATRIX COMPOSITES REFORCED WITH HEMP FIBERS}

\section{Abstract}

Nowadays, the necessity to use environmentally friendly materials is increasing in parallel to the constant search for new technologies to produce of renewable, economically viable and technologically advanced materials, which justifies this work. This present work consists to study the mechanical behavior of polyester matrix composites reinforced with hemp fibers and tested in Izod impact. For the accomplishment of this test, specimens with the dimensions of $10 \mathrm{~mm}$ of width, 63 $\mathrm{mm}$ of length and $12.7 \mathrm{~mm}$ of thickness, were made in different volume fractions of fiber, $0 \%, 10 \%, 20 \%$ and $30 \%$. The samples were tested in a PANTEC pendulum set in Izod configuration, model XC-50 located on LAMAV/UENF. The Izod impact energy of the $30 \%$ polyester composites of hemp fibers was $169,4 \mathrm{~J} / \mathrm{m}$.

Keywords: Izod Impact Test; Polyester; Hemp Fiber.

1 Laboratório de Materiais Avançados (LAMAV); Universidade Estadual do Norte Fluminense (UENF), Campos dos Goytacazes - RJ, Brasil.

2 Faculdade Redentor, Itaperuna - RJ, Brasil.

3 Instituto Militar de Engenharia (IME), Rio de Janeiro - RJ, Brasil.. 


\section{INTRODUÇÃO}

Os compósitos poliméricos são materiais de moldagem estrutural formados por uma fase polimérica contínua chamada matriz, que é reforçada por uma fase descontínua, reforço, que são adicionados físico-quimicamente antes do processo de polimerização (cura). A fase descontínua, ou reforço, geralmente consiste em fibras que podem ser de vidro, aramida, carbono, origem lignocelulósica natural ou animal, dependendo da aplicação final [1]. A fase contínua, a matriz, é geralmente composta por uma resina que pode ser termoplástica ou termofixas [2]. Os materiais compósitos com matriz polimérica são capazes de combinar boa resistência mecânica com boa ductilidade da matriz, oferecendo a possibilidade de obter materiais com as propriedades desejadas [3].

As propriedades finais de um composto dependem diretamente das características das fases constituintes (fibra/matriz), a quantidade relativa e a geometria da fase dispersa (reforço). Além das características físicas e morfológicas das fases constituintes, o desempenho final dos compósitos depende das propriedades mecânicas da matriz e do reforço, e da efetiva interação fibra/matriz [5].

A maioria das matrizes poliméricas usadas em compósitos são resinas termofixas, especialmente resinas epóxi, fenólicas e poliéster. As resinas de poliéster, além de um baixo custo, boa estabilidade dimensional, fácil manuseio, também possuem propriedades mecânicas satisfatórias, o que torna viável como uma matriz polimérica amplamente utilizada na indústria [6-8]. Além disso, a resina epóxi tem um custo maior, mas possui boa resistência química, elétrica e térmica. Devido à sua alta resistência mecânica e química, é amplamente utilizado em laminados especiais, tubulações, tanques, aviões, navios, entre outros [9-11].

Como reforço, o uso de fibras lignocelulósicas está sendo justificado por seu baixo custo, abundância, sustentabilidade, baixa densidade e propriedades mecânicas satisfatórias que tornam essas matérias-primas uma alternativa potencial para fibras sintéticas, como a fibra de vidro [12-13]. As fibras de cânhamo são fibras lignocelulósicas naturais extraídas do caule da Cannabis Sativa, ricas em celulose que apresentam propriedades mecânicas satisfatórias. Para além do seu principal uso em produtos têxteis, essas fibras podem oferecer propriedades relevantes na gama de novos materiais compósitos [14], uma vez que proporcionam melhores resultados em suas propriedades físicas e térmicas, modificam a aparência da superfície e as características de processamento, e reduzem o custo final do material [15].

O teste de impacto é o principal parâmetro para determinar a energia utilizada na deformação e ruptura da amostra. Esse teste envolve submeter a amostra entalhada a uma flexão causada por um martelo de impacto, que pode ser realizado de acordo com duas configurações, Charpy e Izod, diferenciadas da configuração final dos corpos de prova e da maneira como eles estão ligados à máquina de teste [16-17]. De acordo com a norma ASTM 256 [18], os corpos de prova (CPs) de teste de impacto izod têm uma seção quadrada de $10 \mathrm{~mm}$ de lado e um comprimento de 120 $\mathrm{mm}$, com um entalhe em forma de $\mathrm{V}$ no meio das amostras.

Com isso, o presente estudo propõe a análise mecânica dos compostos de matriz poliéster reforçados com fibras de cânhamo 10\%, 20\% e 30\% em volume sob a configuração do teste de impacto izod. Os CPs foram testados em um pêndulo PANTEC, modelo XC-50 localizado em LAMAV / UENF, configurado para teste de impacto izod. Os resultados obtidos permitiram caracterizar esses novos materiais quanto a energia necessária para que haja sua ruptura, contribuindo para o 
processo de busca de novos materiais sustentáveis, economicamente viáveis e tecnologicamente avançados.

\section{MATERIAIS E MÉTODOS}

Para fabricação das amostras, utilizou-se: uma matriz metálica nas dimensões $62 \mathrm{x}$ 12,7 x 10 mm Fig. 1a; e as fibras de cânhamo, Fig. 1b, foram adquiridas da Desigan Fibras Naturais, empresa que comercializa fibras naturais; a matriz polimérica foi a resina poliéster ortoftálica com $0,5 \%$ de iniciador à base de metiletilcetona.
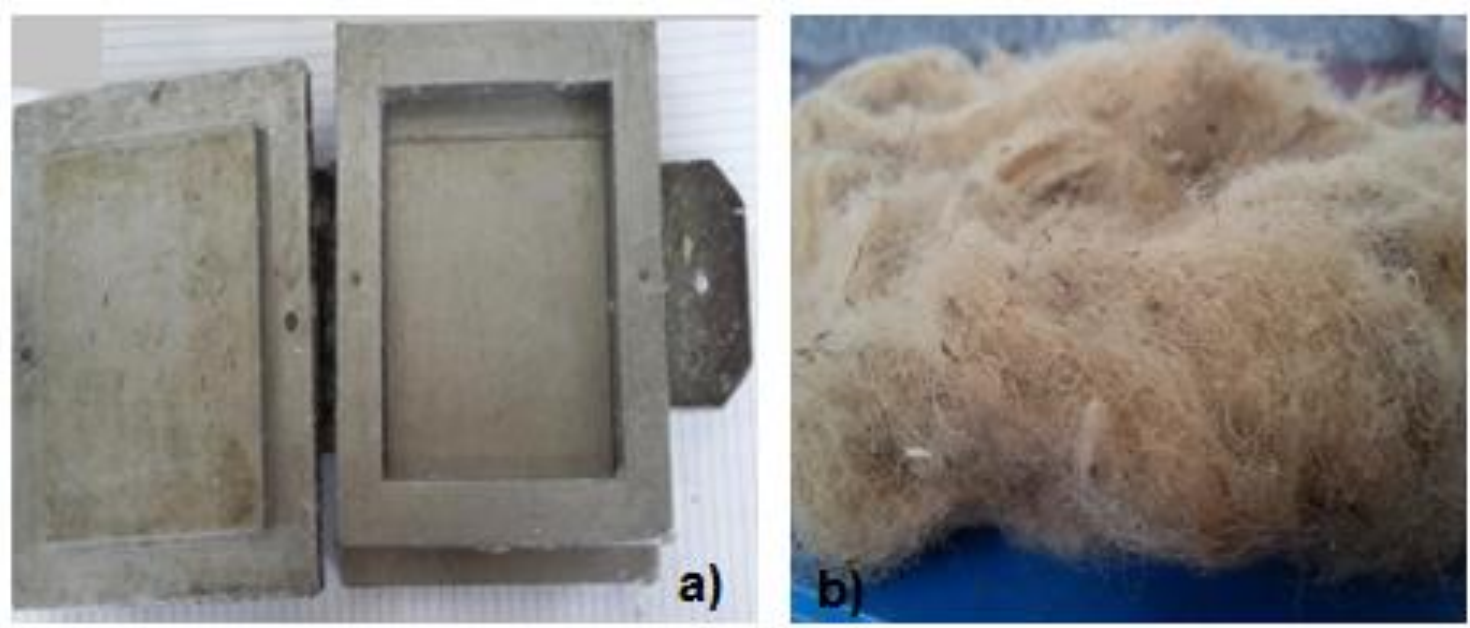

Figura 1. a) Matriz de metal usada para moldar os CPs para o teste de impacto izod e b) Aspecto da fibra de cânhamo.

\subsection{Caracterização Dimensional da Fibra de Cânhamo}

A fibra de cânhamo foi caracterizada pelo seu diâmetro e comprimento. Inicialmente, 100 fibras foram selecionadas aleatoriamente do lote. Estas tiveram seu comprimento medido com a ajuda de um paquímetro. Seus diâmetros foram obtidos através de um projetor de perfil Nikon modelo 6C no LAMAV/UENF, mostrado na Fig. 2.

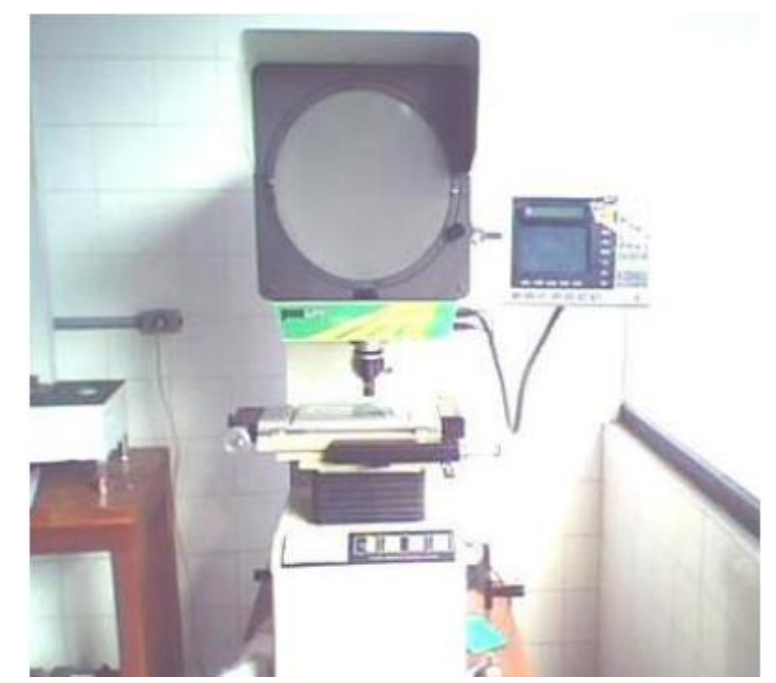

Figure 2. Projetor de perfil Nikon, usado para medir os diâmetros das fibras. 
Cada fibra teve seu diâmetro medido individualmente no projetor de perfil em cinco pontos distintos ao longo do seu comprimento. Para cada um desses pontos, o diâmetro foi medido a $0^{\circ}$ e após a rotação de $90^{\circ}$, para obter as mais variadas dimensões possíveis. No final, o diâmetro e o comprimento foram analisados estatisticamente.

As fibras de cânhamo foram alinhadas de forma continua na matriz de aço, junto com a resina poliéster fluida, além de seu endurecedor específico. Após a vedação da matriz, o período de cura foi realizado durante 24 horas sob pressão, à temperatura ambiente. Após o processo de cura, as placas produzidas a partir de cada compósito foram cortadas de acordo com a norma ASMT D256 [17], com 10 $\mathrm{mm}$ de largura, $63 \mathrm{~mm}$ de comprimento e $12,7 \mathrm{~mm}$ de espessura na direção do alinhamento das fibras da amostras. O entalhe padrão, para testes de impacto Izod, foram feitos de acordo com a norma ASTM D256 [17], profundidade de 2,54 mm, ângulos de $45^{\circ}$ e um raio de curvatura da ponta de 0,25, Fig. 3b. Para cada condição, foram testados 10 espécimes para assegurar uma validação estatística. As amostras foram testadas por impacto em um pêndulo PANTEC com configuração Izod, Figura 3a. A energia de impacto foi obtida usando um martelo de potência de $11 \mathrm{~J}$ para compósitos com 0, 10\%, 20\%, 30\% de fibra.
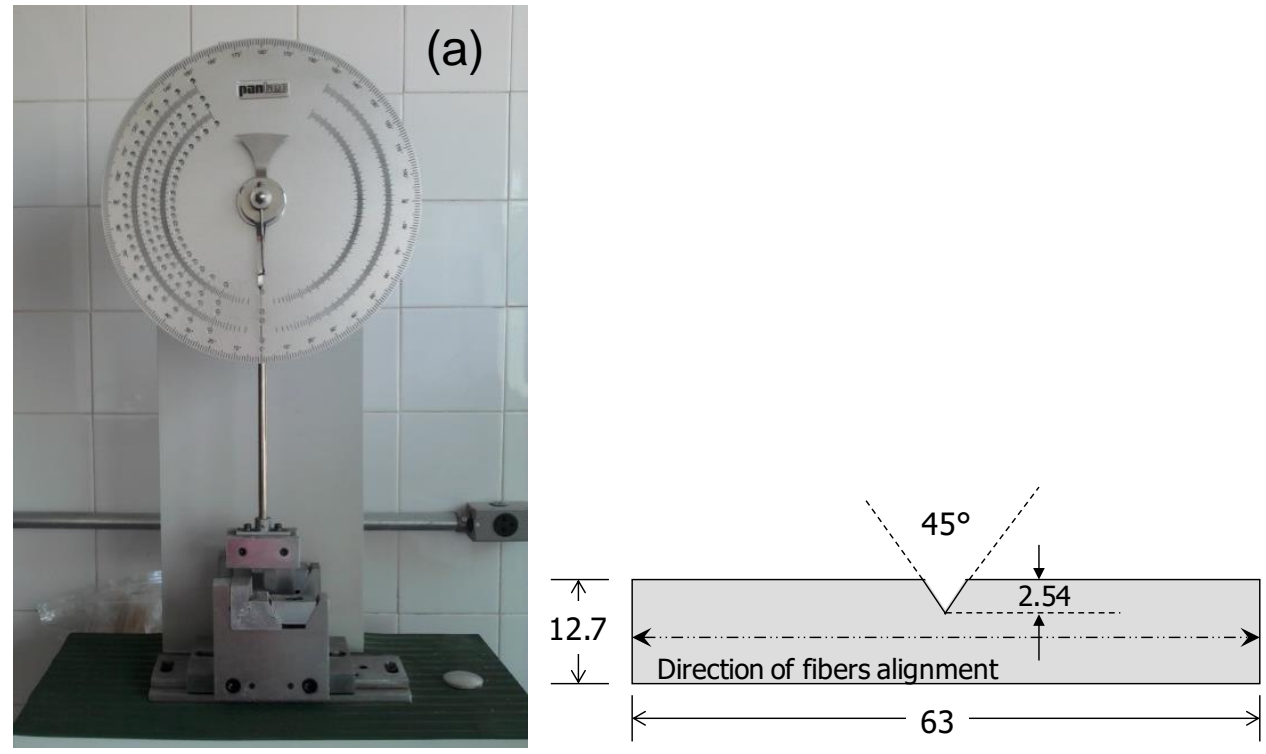

(b)

Figura 3. a) PANTEC, equipamento para teste de impacto Izod e b) esquemática de corpo de prova padrão para teste de impacto Izod.

\section{RESULTADOS E DISCUSSÃO}

A Tabela 1 apresenta os resultados dos testes de impacto Izod de compósitos de matriz poliéster reforçados com diferentes frações volumétricas de fibras de cânhamo. 
Tabela 1. Energia de impacto Izod para compósitos de poliéster reforçados com fibras de cânhamo

\begin{tabular}{cc}
$\begin{array}{c}\text { Fração } \\
\text { Volumétrica de } \\
\text { Fibra de } \\
\text { Cânhamo (\%) }\end{array}$ & $\begin{array}{c}\text { Energia de } \\
\text { Impacto Izod(J/m) }\end{array}$ \\
\hline 0 & $21,8 \pm 9,7$ \\
\hline 10 & $60,2 \pm 14,7$ \\
\hline 20 & $111,9 \pm 23,3$ \\
\hline 30 & $169,4 \pm 29,2$ \\
\hline
\end{tabular}

Com base nos resultados apresentados na Tabela 1, a variação da energia de impacto Izod com a quantidade de fibra de cânhamo no compósito de poliéster é mostrada na Fig. 4.

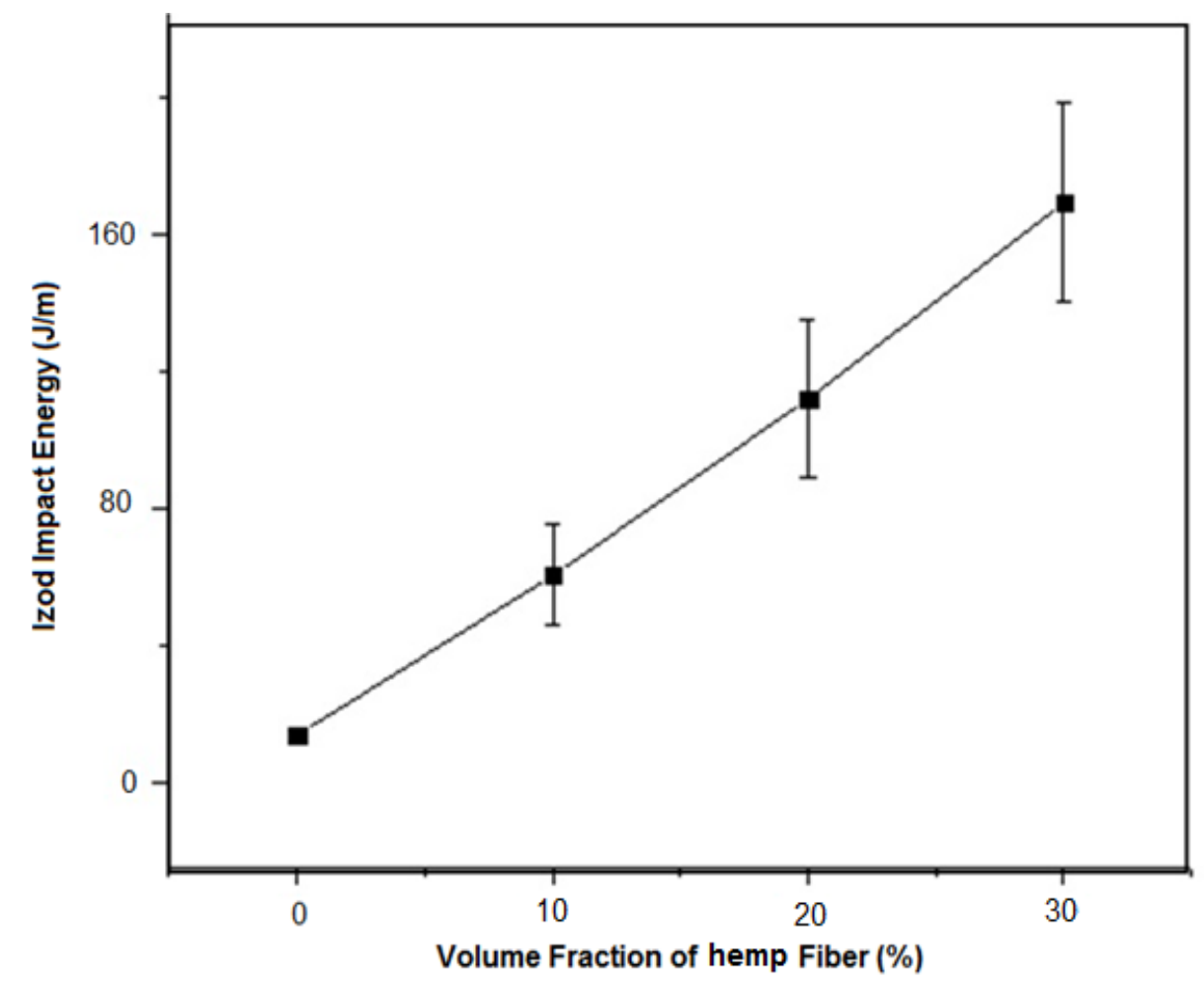

Figura 4. Energia de impacto Izod em função de diferentes frações volumétricas da fibra de cânhamo.

Nesta figura, deve-se notar que a incorporação da fibra de cânhamo na matriz melhora significativamente a resistência ao impacto do compósito. Esta melhoria pode ser considerada quase como um aumento exponencial em relação à quantidade de fibras de cânhamo.

A alta dispersão relativa de valores, dada pelo desvio padrão associado aos pontos de percentagens mais elevadas de fibras na Fig. 4, se deve a característica heterogênea das fibras lignocelulósicas [11]. O reforço de uma matriz polimérica com fibras sintéticas [13-14] e naturais [15-16] aumentam a resistência ao impacto do compósito.

A interação de interface relativamente fraca entre uma fibra natural hidrofílica e uma matriz polimérica hidrofóbica, contribui para uma transferência de carga ineficaz da 
matriz para a fibra mais longa. Essa característica permite que o sistema absorva mais energia devido à flexibilidade da fibra que se desliza para fora da matriz, mas não quebra, o que amplifica a energia necessária para romper o espécime [17]. Os aspectos macroscópicos típicos dos CPs quebrados pelos testes de impacto Izod são mostrados na Fig. 5. Nesta figura, deve-se notar que a incorporação de fibras resulta em uma forma de ruptura completamente diferente em relação ao poliéster puro $(0 \%$ de fibra) em que ocorre uma ruptura totalmente transversal.

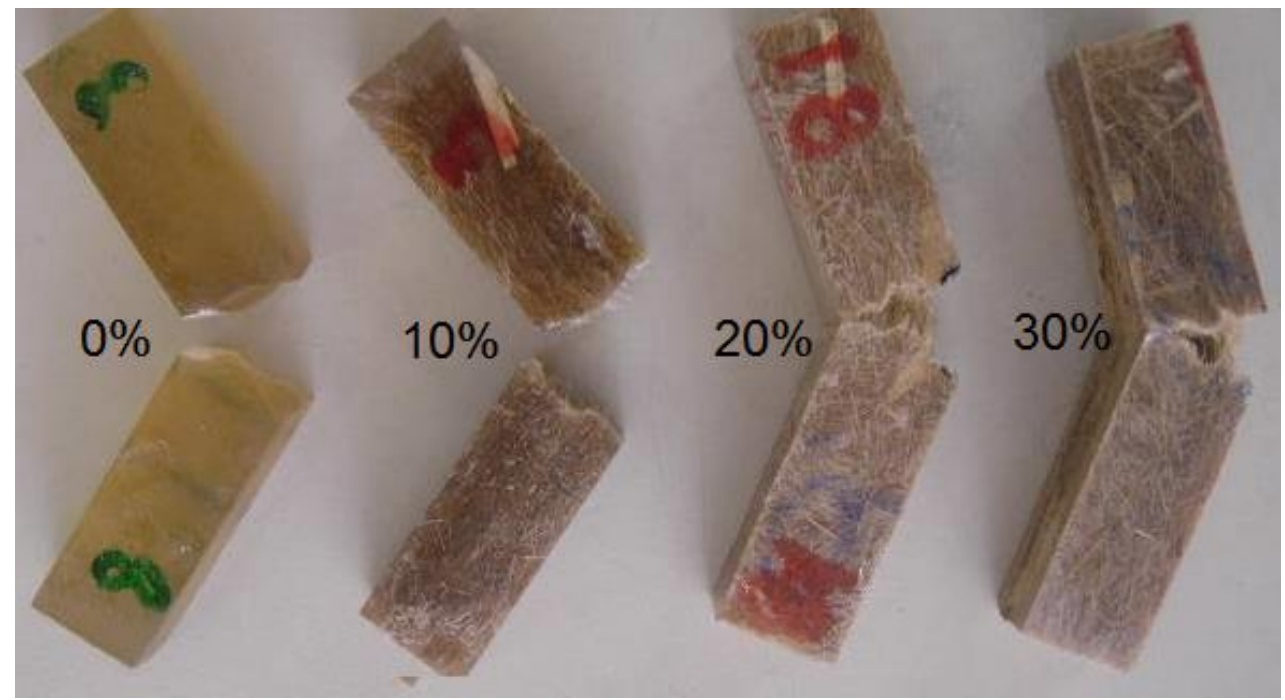

Figura 5. CPs tipicamente rompidos de compósitos de poliéster reforçados com fibras de cânhamo por testes de impacto Izod.

Com $30 \%$ de fibra, a ruptura não é mais completamente transversal. Isto indica que a trinca nucleada no entalhe se propaga inicialmente transversal através da matriz, conforme esperado em um polímero monolítico, no entanto, quando a trinca atinge uma fibra, a ruptura irá mudar de direção e precorrer um novo caminho, que que será a interface fibra/matriz. Como consequência, após o martelo atingiu o $\mathrm{CP}$, algumas fibras longas serão retiradas da matriz, mas não serão quebradas, simplesmente se curvarão. De fato, para as frações volumétricas de fibras de $30 \mathrm{vol} \%$, alguns espécimes não estão separados. Para essas quantidades de fibras, parte da amostra foi dobrada o suficiente para permitir que o martelo continuasse sua trajetória levando o espécime sem quebrá-lo em pedaços, o que é esperado em um teste de Izod. O valor da resistência ao impacto neste caso não pode ser comparado com outros em que o espécime é totalmente separado. De qualquer forma, o fato de um espécime não estar completamente separado em duas partes subestima a resistência ao impacto. Em outras palavras, se todas as fibras estavam quebradas, a energia de impacto adsorvido seria ainda maior.

\section{CONCLUSÃO}

- Existe um aumento significativo na energia absorvida nos testes de impacto Izod com a incorporação de fibras de cânhamo em um compósito de matriz de poliéster.

- A interface fraca entre as fibras de cânhamo e a matriz de poliéster contribui muito para aumentar a energia de impacto alterando a trajetória de fissuras no compósito. 
- A maior parte desse aumento da tencidad é, aparentemente, devido ao baixo risco de cisalhamento interfacial da fibra/poliéster. Isso resulta em uma energia absorvida maior, como conseqüência de uma propagação longitudinal das rachaduras ao longo da interface, o que gera áreas de ruptura maiores, em comparação com uma fratura transversal.

\section{AGRADECIMENTOS}

Os autores agradecem o apoio a esta investigação pelas agências brasileiras: CNPq, CAPES e FAPERJ.

\section{REFERÊNCIAS}

[1] Aquino RGMP, Almeida JRM, Monteiro SN. Análise do compósito de piaçava/ resina poliéster, como substituto da madeira. In: 60ํㅡㄹ Congresso Anual da Associação Brasileira de Metalurgia e Materiais- ABM, Belo Horizonte-MG. 2005; 1484-1492.

[2] Adamian R, Medina V, Weisz J. Novos materiais: tecnologia e aspectos econômicos. Rio de Janeiro: COOPE- UFRJ; 2009.

[3] Monteiro SN, Rodrigues RJS, Souza MVE, D'Almeida JRM. Sugar cane bagassewaste as reinforcement in low cost composites. Adv. Performance Materials. 1998; 5:183-191.

[4] Ventura AMFM. Os Compósitos e a sua aplicação na Reabilitação de Estruturas metálicas Ciência \& Tecnologia dos Materiais. 2009; 21(3/4): 10-19.

[5] Hage Jr E. Compósitos e Blendas Poliméricas, Campinas: Instituto Latino Americano de Tecnologia e IBM; 1989.

[6] Goodman SH. Handbook of Thermoset Plastics. 2. ed. New Jersey: Noyes Publications; 1998.

[7] Mallick PK. Fiber Reinforced Composites. New York; 1993.

[8] Bledzki AK, Gassan J. Composites reinforced with cellulose based fibres. Progress Polymer Science. 1999; 24: 221-274.

[9] Strong AB. Fundamental of composite: Materials, Methods, and applications. Dearborn, Michigan: Society of Manufacturing Engineeres; 1989.

[10] Rosato DV, Rosato DV. Reinforced plastics Handbook. Publisher: Elsevier; 2004.

[11] Da Silva RV, Aquino EMF, Rodrigues LPS, Barros ARF. Desenvolvimento de um compósito laminado híbrido com fibras natural e sintética. Revista Matéria.2008; 13(1): 154-161.

[12] Pacheco EB, Santos MS, Dias ML. Materiais reciclados á base de PET a cargas de coco. Brasil; 2000.

[13] Crônier D, Monties B, Chabbert B. Structure and Chemical Composition of Bast Fibers Isolated from Developing Hemp Stem. Journal of Agricultural and Food Chemistry. 2005; 53: 8279-8289.

[14] Lavengood RE, Silver FM. Interpretating supplier data sheets. Engineering Materials Handbook- Engineering Plastic: Metals Park: ASM International.1988; 2: 638-645.

[15] Souza SA. Ensaios Mecânicos de Materiais Metálicos:Fundamentos Teóricos e Práticos. São Paulo: Edgard BlucherLtda; 1982.

[16] Fu SY, Lauke B, Mäder E, Hu X, Yue CY. Fracture Resistance of Short-GlassFiber-Reinforced and Short-Carbon-Fiber-Reinforced Poly-propylene under 
Charpy Impact Load and its Dependence on Processing. Journal of Materials Processing Technologies. 1999; 89/90: 501-507.

[17] ASTM, Internacional (2002) Standard Test Methods for Determining the Izod Impact Resistance of Notched Specimens of Plastic: ASTM D 256. United States. 